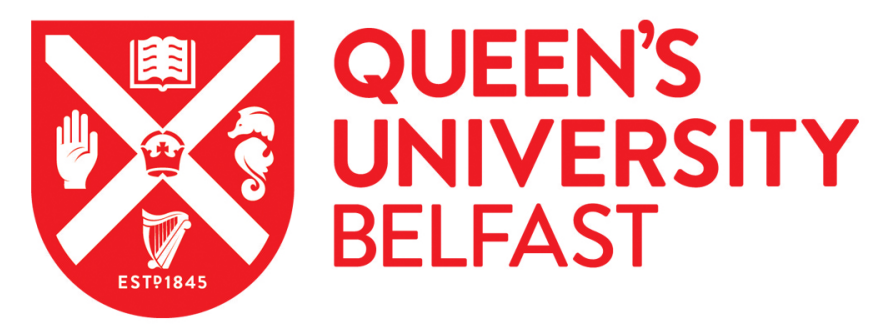

\title{
On controlled-invariance and stabilization of time-delay systems
}

Athanasopoulos, N., \& Lazar, M. (2014). On controlled-invariance and stabilization of time-delay systems. In 2014 European Control Conference (pp. 778-783). Institute of Electrical and Electronics Engineers Inc.. https://doi.org/10.1109/ECC.2014.6862474

\section{Published in:}

2014 European Control Conference

\section{Document Version:}

Peer reviewed version

Queen's University Belfast - Research Portal:

Link to publication record in Queen's University Belfast Research Portal

\section{Publisher rights}

Copyright 2014 EUCA

This work is made available online in accordance with the publisher's policies. Please refer to any applicable terms of use of the publisher.

\section{General rights}

Copyright for the publications made accessible via the Queen's University Belfast Research Portal is retained by the author(s) and / or other copyright owners and it is a condition of accessing these publications that users recognise and abide by the legal requirements associated with these rights.

Take down policy

The Research Portal is Queen's institutional repository that provides access to Queen's research output. Every effort has been made to ensure that content in the Research Portal does not infringe any person's rights, or applicable UK laws. If you discover content in the Research Portal that you believe breaches copyright or violates any law, please contact openaccess@qub.ac.uk. 


\title{
On Controlled-Invariance and Stabilization of Time-Delay Systems
}

\author{
Nikolaos Athanasopoulos ${ }^{\star}$ and Mircea Lazar
}

\begin{abstract}
A non-conservative synthesis approach for discrete-time linear time-delay systems is presented, induced by a relaxed notion of invariance, namely, controlled $(k, \lambda)-$ $D$-contractiveness. We characterize two particular families of controlled $(k, \lambda)$-contractive sets in the augmented state space of the corresponding delay-free system and establish their relationship to controlled $(k, \lambda)-D$-contractive sets of the timedelay system. The required computations for both the controller synthesis and controller implementation are carried in the state space of the time-delay system, making the method scalable with respect to the maximum delay.
\end{abstract}

\section{INTRODUCTION}

The stability analysis and controller synthesis of timedelay systems remains an important and challenging problem [1]-[3]. There are two main Lyapunov based approaches towards the stabilization of linear time-delay systems, see e.g. [4], [5] for an overview. The first approach induces Lyapunov-Krasovskii functions, see e.g. [6], and requires the augmentation of the state vector with the delayed states and inputs, resulting in a delay-free discrete-time linear system. Following this approach, stabilizing controllers can be computed by applying, with modifications, results from control theory [6]-[9]. The second approach [10]-[14] deals directly with the time-delay system by employing LyapunovRazumikhin functions [15, Chapter 5.4]. The methods that belong to the first category are non-conservative in obtaining stabilizing control laws. However, they do not scale well with respect to the maximum delay. On the other hand, the second approach is limited by conservatism since it is based on sufficient only conditions for stabilization. The recent work [14] offers necessary and sufficient conditions for stability of time-delay systems via a Razumikhin-type approach, which however, have not been exploited for synthesis.

Regarding set invariance, the Krasovskii approach induces contractive sets with respect to the closed-loop augmented system. Unfortunately, these sets do not necessarily induce a contractive set in the original state space. On the other hand, the Razumikhin approach, whenever successful in obtaining a stabilizing control law, always leads to the characterization of contractive sets in the original state space of the time-delay system [16], [17]. Such invariant sets have been recently named as $D$-invariant sets [18]. Other notions of set invariance can be found in [19], [20]. A recent relaxation of set invariance is the $(k, \lambda)$-contractiveness and

\footnotetext{
${ }^{\star}$ Supported by the People Programme (Marie Curie Actions) of the European Union's Seventh Framework Programme (FP7/2007-2013 under REA grant agreement $\mathrm{n}^{\circ} 302345$ ).

The authors are with the Eindhoven University of Technology, Den Dolech 2, 5600MB, The Netherlands. E-mail addresses: n.athanasopoulos@tue.nl, m.lazar@tue.nl
}

controlled $(k, \lambda)$-contractiveness [21], [22]. For discretetime homogeneous systems, the sets having these properties are also regions of attraction or regions of stabilizability. Moreover, differently from the classical notions of positive and controlled invariance, any convex and compact set that includes the origin in its interior is a controlled $(k, \lambda)-$ contractive set. This is very relevant for the time-delay case, because it allows to characterize controlled $(k, \lambda)$-contractive sets in the augmented state space which induce controlled $(k, \lambda)-D$-contractive sets in the original state space by projection.

The main goal of this article is to provide a scalable and non-conservative method of simultaneously computing a region of attraction and a stabilizing state-feedback control law for the time-delay system. To this end, we identify suitable families of controlled $(k, \lambda)$-contractive proper $\mathcal{C}-$ polytopic sets in the augmented state space. These sets induce the controlled $(k, \lambda)-D$-contractiveness property to a proper $\mathcal{C}$-polytopic set defined in the original state space. The required computations for the characterization of controlled$(k, \lambda)-D$-contractive sets are carried out in the original state space, rendering the proposed method scalable. The obtained stabilizing controller is a scalable vertex-interpolation based control law. The proposed method can be modified to handle state and input constraints.

In Section II, the necessary definitions and a few preliminaries are given. In Section III the main results are presented. The efficiency of the proposed method is illustrated in a numerical example in Section IV, while conclusions are drawn in Section V.

\section{PRELIMINARIES AND BASIC DEFINITIONS}

Let $\mathbb{R}, \mathbb{R}_{+}, \mathbb{Z}$ and $\mathbb{N}$ denote the field of real numbers, the set of non-negative reals, the ring of integers and the set of nonnegative integers, respectively. For every $c \in \mathbb{R}$ and $\Pi \subseteq \mathbb{R}$ we define $\Pi_{\geq c}:=\{k \in \Pi \mid k \geq c\}$, and similarly $\Pi_{\leq c}, \mathbb{R}_{\Pi}:=\Pi$ and $\mathbb{N}_{\Pi}:=\mathbb{N} \cap \Pi$. For a matrix $A \in \mathbb{R}^{n \times m}$, $[A]_{i j}$ denotes the element in the $i$-th row and $j$-th column, $[A]_{i:} \in \mathbb{R}^{m}$ denotes the $i$-th row and $[A]_{: j} \in \mathbb{R}^{n}$ denotes the $j$-th column. Given a vector $x \in \mathbb{R}^{n},[x]_{i} \in \mathbb{R}$ denotes the $i$-th entry of $x$. Given two integers $c, d$ such that $c<d$ and vectors $x_{i} \in \mathbb{R}^{n}, i \in \mathbb{N}_{[c, d]}$, the ordered vector sequence $\left\{x_{c}, x_{c+1}, \ldots, x_{d-1}, x_{d}\right\}$ is denoted by $\boldsymbol{x}_{[c, d]}$. The identity matrix is denoted by $I_{n} \in \mathbb{R}^{n \times n}$, the zero matrix is denoted by $0_{n \times m} \in \mathbb{R}^{n \times m}$ and the vector with all elements equal to one is denoted by $1_{n} \in \mathbb{R}^{n}$. A proper $C$-set $\mathcal{S} \subset \mathbb{R}^{n}$ is a compact, convex set which contains the origin in its interior. Given a set $\mathcal{S} \subset \mathbb{R}^{n}$ and a real scalar $\alpha \in \mathbb{R}$, the set $\alpha \mathcal{S}$ is defined by $\alpha \mathcal{S}:=\left\{x \in \mathbb{R}^{n}:(\exists y \in \mathcal{S}\right.$ : 
$x=\alpha y)\}$. A polytope is the bounded intersection of a finite number of closed half-spaces. Proper $\mathcal{C}$-polytopic sets are described by half-space or vertex representations. The vertex representation of an arbitrary proper $\mathcal{C}$-polytopic set $\mathcal{S}$ corresponds to

$$
\mathcal{S}:=\operatorname{conv}\left(\left\{v^{i}\right\}_{i \in \mathbb{N}_{[1, q]}}\right),
$$

for some $q \in \mathbb{N}_{\geq n+1}$. We define with $V:=\left[v_{1}, v_{2}, \ldots, v_{q}\right] \in$ $\mathbb{R}^{n \times q}$ the full row-rank matrix that has as columns the vertices of $\mathcal{S}$. Given a proper $C$-set $\mathcal{S} \subset \mathbb{R}^{n}$, the function

$$
\Psi(\mathcal{S}, x):=\inf _{\mu}\{\mu: x \in \mu \mathcal{S}, \mu \geq 0\},
$$

defined for any $x \in \mathbb{R}^{n}$, is called the Minkowski function of the set $\mathcal{S}$, or the gauge function of the set $\mathcal{S}$. The function $\phi(\cdot): \mathbb{R}_{+} \rightarrow \mathbb{R}_{+}$belongs to class $\mathcal{K}$ if it is continuous, strictly increasing and $\phi(0)=0$. The function $\beta: \mathbb{R}_{+} \times$ $\mathbb{R}_{+} \rightarrow \mathbb{R}_{+}$belongs to class $\mathcal{K} \mathcal{L}$ if for each fixed $t \in \mathbb{R}_{+}$, $\beta(\cdot, t) \in \mathcal{K}$, and for each fixed $s \in \mathbb{R}_{+}, \beta(s, \cdot)$ is decreasing and $\lim _{t \rightarrow \infty} \beta(s, t)=0$. The map $g(\cdot): \mathbb{R}^{n} \rightarrow \mathbb{R}^{m}$ is called a positively homogeneous map of order one in a set $\mathcal{Z} \subset \mathbb{R}^{n}$, or simply homogeneous in $\mathcal{Z}$, if for any scalar $\alpha \in \mathbb{R}_{+}$and any vector $z$ such that $\alpha z \in \mathcal{Z}$ it holds that $g(\alpha z)=\alpha g(z)$. If $\mathcal{Z}=\mathbb{R}^{n}$, the map $g(\cdot)$ is called homogeneous.

We consider linear discrete-time systems with maximum delay $N \in \mathbb{N}$, described by the difference equation

$$
x_{t+1}=\sum_{i=0}^{N} A_{i} x_{t-i}+B u_{t},
$$

where $x \in \mathbb{R}^{n}$ is the state vector, $u \in \mathbb{R}^{m}$ is the input vector, $A_{i} \in \mathbb{R}^{n \times n}, i \in \mathbb{N}_{[0, N]}, B \in \mathbb{R}^{n \times m}$ are the system matrices and $t \in \mathbb{N}$ is the time variable. The initial condition of system (2) is a vector sequence $\boldsymbol{x}_{[-N, 0]}$ of length $N+1$. We denote the dynamics of (2) by $\Phi(\cdot, \cdot): \mathbb{R}^{n(N+1)} \times \mathbb{R}^{m} \rightarrow \mathbb{R}^{n}$. Setting the stacked vector $z \in \mathbb{R}^{n(N+1)}$ to contain the $N+1$ state vectors of system (2), i.e.,

$$
z_{t}:=\left[\begin{array}{llll}
x_{t}^{\top} & x_{t-1}^{\top} & \ldots & x_{t-N}^{\top}
\end{array}\right]^{\top},
$$

we define the delay-free augmented discrete-time linear system with the difference equation

$$
z_{t+1}=A_{z} z_{t}+B_{z} u_{t}
$$

where $A_{z} \in \mathbb{R}^{n(N+1) \times n(N+1)}, B_{z} \in \mathbb{R}^{n(N+1) \times m}$ are the corresponding system matrices, i.e.,

$$
A_{z}:=\left[\begin{array}{ccccc}
A_{0} & A_{1} & \ldots & A_{N-1} & A_{N} \\
I_{n} & 0_{n \times n} & \ldots & 0_{n \times n} & 0_{n \times n} \\
\vdots & \vdots & \ddots & \vdots & \vdots \\
0_{n \times n} & 0_{n \times n} & \ldots & I_{n} & 0_{n \times n}
\end{array}\right],
$$

$B_{z}:=\left[\begin{array}{ll}B^{\top} & 0_{m \times n} \ldots 0_{m \times n}\end{array}\right]^{\top}$ and $t \in \mathbb{N}$ is the time variable. The subvectors $\left[z_{t}\right]_{i} \in \mathbb{R}^{n}, i \in \mathbb{N}_{[1, N+1]}$ of the augmented state space $z \in \mathbb{R}^{n(N+1)}$ are defined as

$$
\left[z_{t}\right]_{i}:=x_{t+1-i}, \quad i \in \mathbb{N}_{[1, N+1]}, \quad \text { for all } t \in \mathbb{N} .
$$

We consider the class of state-feedback control laws $g(\cdot)$ : $\mathbb{R}^{n(N+1)} \rightarrow \mathbb{R}^{m}$ for both systems (2) and (4). The closedloop dynamics of (2), denoted by $\Phi_{x}(\cdot): \mathbb{R}^{n(N+1)} \rightarrow \mathbb{R}^{n}$, is equal to

$$
\Phi_{x}\left(\boldsymbol{x}_{[-N, 0]}\right):=\sum_{i=0}^{N} A_{i} x_{-i}+B g\left(\boldsymbol{x}_{[-N, 0]}\right) .
$$

Then, the $k$-th iterated map $\Phi_{x}^{k}\left(\boldsymbol{x}_{[-N, 0]}\right)$ of the closed-loop dynamics is defined as follows. For $k=0$, it holds that $\Phi_{x}^{0}\left(\boldsymbol{x}_{[-N, 0]}\right):=x_{0}$. For $k=1$, it holds that $\Phi_{x}^{1}\left(\boldsymbol{x}_{[-N, 0]}\right):=$ $\Phi_{x}\left(\boldsymbol{x}_{[-N, 0]}\right)$, as defined in (6). For $k \in \mathbb{N}_{[2, N+1]}$, it is

$\Phi_{x}^{k}\left(\boldsymbol{x}_{[-N, 0]}\right):=\Phi_{x}\left(\boldsymbol{x}_{[-N+k-1,0]} \cup\left\{\Phi_{x}^{i}\left(\boldsymbol{x}_{[-N, 0]}\right)\right\}_{i \in \mathbb{N}_{[1, k-1]}}\right)$, while for $k \in \mathbb{N}_{>N+1}$,

$$
\Phi_{x}^{k}\left(\boldsymbol{x}_{[-N, 0]}\right):=\Phi_{x}\left(\left\{\Phi_{x}^{i}\left(\boldsymbol{x}_{[-N, 0]}\right)_{i \in \mathbb{N}_{[k-N-1, k-1]}}\right\}\right) .
$$

Similarly, the closed-loop dynamics of (4), denoted by $\Phi_{z}(\cdot): \mathbb{R}^{n(N+1)} \rightarrow \mathbb{R}^{n(N+1)}$, is equal to

$$
\Phi_{z}(z)=A_{z} z+B_{z} g(z)
$$

while the $k$-th iterated map is given by $\Phi_{z}^{k}(z):=$ $\Phi_{z}\left(\Phi_{z}^{k-1}(z)\right)$, for any $k \in \mathbb{N}_{\geq 1}$. By convention, $\Phi_{z}^{0}(z):=z$.

Definition 1 The closed-loop system (7) is called $\mathcal{K} \mathcal{L}-$ stable in a set $\mathcal{Z} \subset \mathbb{R}^{n(N+1)}$ if there exists a $\mathcal{K} \mathcal{L}$-function $\beta(\cdot, \cdot)$ such that for all $z_{0} \in \mathcal{Z}$ the state trajectory of the closed-loop system (7) satisfies the relation

$$
\left\|z_{t}\right\| \leq \beta\left(\left\|z_{0}\right\|, t\right), \quad \forall t \in \mathbb{N} .
$$

Definition 2 The closed-loop system (6) is called $\mathcal{K} \mathcal{L}$ stable in a set $\mathcal{S} \subset \mathbb{R}^{n}$ if there exists a $\mathcal{K} \mathcal{L}$-function $\beta(\cdot, \cdot)$ such that for all $x_{i} \in \mathcal{S}, i \in \mathbb{N}_{[-N, 0]}$, the state trajectory of the closed-loop system (6) satisfies the relation

$$
\left\|x_{t}\right\| \leq \beta\left(\left\|\boldsymbol{x}_{[-N, 0]}\right\|, t\right), \quad \forall t \in \mathbb{N} .
$$

In the above definitions if $\mathcal{Z}=\mathbb{R}^{n(N+1)}$ and $\mathcal{S}=\mathbb{R}^{n}$, the closed-loop systems (7) and (6) are called globally $\mathcal{K} \mathcal{L}-$ stable. The notions of controlled $(k, \lambda)$-contractive sets [22] are recalled next.

Definition 3 Given a real scalar $\lambda \in \mathbb{R}_{[0,1]}$ and an integer $k \in \mathbb{N}_{\geq 1}$, the proper $\mathcal{C}$-set $\mathcal{Z} \subset \mathbb{R}^{n(N+1)}$ is called a controlled $(k, \lambda)$-contractive set with respect to system (4) if and only if there exists a state-feedback control law $g(\cdot): \mathbb{R}^{n(N+1)} \rightarrow \mathbb{R}^{m}$ such that for all $z \in \mathcal{Z}$ it holds that $\Phi_{z}^{k}(z) \in \lambda \mathcal{Z}$.

If the condition in Definition 3 is satisfied for $\lambda=1$, the set $\mathcal{Z}$ is called a controlled $(k, 1)$-invariant set. Next, the corresponding notions of the controlled $(k, \lambda)$-contractive sets for time-delay systems of the form (2) are presented.

Definition 4 Given a real scalar $\lambda \in \mathbb{R}_{[0,1]}$ and an integer $k \in \mathbb{N}_{\geq 1}$, the proper $\mathcal{C}-$ set $\mathcal{S} \subset \mathbb{R}^{n}$ is called a controlled $(k, \lambda)-D$-contractive set with respect to system (2) if and 
only if there exists a state-feedback control law $g(\cdot)$ : $\mathbb{R}^{n(N+1)} \rightarrow \mathbb{R}^{m}$ such that for all $x_{i} \in \mathcal{S}, i \in \mathbb{Z}_{[-N, 0]}$, it holds that $\Phi^{k+i}\left(\boldsymbol{x}_{[-N, 0]}\right) \in \lambda \mathcal{S}$, for all $i \in \mathbb{Z}_{[-N, 0]}$.

If the condition in Definition 4 is satisfied for $\lambda=1$, the set $\mathcal{S}$ is called a controlled $(k, 1)-D$-invariant set. Notice that the definitions of a controlled $\lambda$-contractive set and of a controlled $\lambda-D-$ contractive set [4] are recovered in both Definitions 3 and 4 for $k=1$. The corresponding notions of $(k, \lambda)$-contractive sets [21] and $D$-contractive sets [4] can be similarly defined for the autonomous case, i.e., when $u_{t}:=0$ in (2), (4), for all $t \in \mathbb{N}$.

\section{MAIN RESULTS}

We make the natural assumption that the matrix pair $\left(A_{z}, B_{z}\right)$ of the augmented system (4) is stabilizable, which is also necessary and sufficient for the system (2) to admit a stabilizing control law [5]. The first problem to be investigated concerns the systematic characterization of any candidate proper $\mathcal{C}$-set $\mathcal{S} \subset \mathbb{R}^{n}$ as a controlled $(k, \lambda)-D$ contractive set and the determination of a stabilizing statefeedback control law for all initial conditions $\boldsymbol{x}_{[-N, 0]}$, where $x_{i} \in \mathcal{S}, i \in \mathbb{N}_{[-N, 0]}$. The second problem concerns the computation of scalable, both in aspects of controller synthesis and implementation, globally stabilizing state-feedback control laws for the time-delay system (2).

A. Characterization of controlled $(k, \lambda)$-contractive sets and induced globally stabilizing control laws

In this section we show that the characterization of a controlled $(k, \lambda)$-contractive set induces globally stabilizing state-feedback controllers for the discrete-time linear augmented system.

Fact 1 Let $\mathcal{Z} \subset \mathbb{R}^{n(N+1)}$ be a proper $\mathcal{C}$-set. Then, for any $z \in \mathbb{R}^{n(N+1)}, z \in \Psi(\mathcal{Z}, z) \mathcal{Z}$.

Fact 2 Let $\mathcal{Z} \subset \mathbb{R}^{n(N+1)}$ be a proper $\mathcal{C}$-set. Then, for all $\alpha \in \mathbb{R}_{+}$, for all $z \in \mathbb{R}^{n(N+1)}, \Psi(\mathcal{Z}, \alpha z)=\alpha \Psi(\mathcal{Z}, z)$.

Proposition 1 Consider the proper $\mathcal{C}$-set $\mathcal{Z} \subset \mathbb{R}^{n(N+1)}$ and let $g(\cdot): \mathcal{Z} \rightarrow \mathbb{R}^{m}$, be a homogeneous control law in $\mathcal{Z}$, such that $\mathcal{Z} \subset \mathbb{R}^{n(N+1)}$ is a $(k, \lambda)$-contractive set with respect to system (7). Consider the homogeneous control law $\bar{g}(\cdot)$ : $\mathbb{R}^{n(N+1)} \rightarrow \mathbb{R}^{m}$ such that $\bar{g}(z):=g(z)$, for all $z \in \mathcal{Z}$. Then, the system $z_{t+1}=\Phi_{\bar{z}}\left(z_{t}\right)$, where $\Phi_{\bar{z}}(\cdot): \mathbb{R}^{n(N+1)} \rightarrow$ $\mathbb{R}^{n(N+1)}$,

$$
\Phi_{\bar{z}}(z):=A z+B \bar{g}(z),
$$

is globally $\mathcal{K} \mathcal{L}$-stable.

The result follows from the observing that the closed-loop system (8) is homogeneous and [21, Theorem V.4], and is omitted here for brevity.

Fact 3 Let $\mathcal{Z} \subset \mathbb{R}^{n(N+1)}$ be a proper $\mathcal{C}$-set. Let $g(\cdot): \mathcal{Z} \rightarrow$ $\mathbb{R}^{n(N+1)}$ be a homogeneous map in $\mathcal{Z}$ and $\bar{g}(\cdot): \mathbb{R}^{n(N+1)} \rightarrow$ $\mathbb{R}^{n(N+1)}$ a map defined by

$$
\bar{g}(z):=\Psi(\mathcal{Z}, z) g\left(\Psi^{-1}(\mathcal{Z}, z) z\right)
$$

for all $z \in \mathcal{Z} \backslash\{0\}$ and $\bar{g}(0):=0$. Then, $\bar{g}(z)=g(z)$, for all $z \in \mathcal{Z}$ and, moreover, $\bar{g}(\cdot)$ is a homogeneous map.

In [22, Proposition 1], it was shown that for stabilizable discrete-time linear systems (4), there always exists a finite integer $k \in \mathbb{N}$ such that any proper $\mathcal{C}$-polytopic set $\mathcal{Z} \subset$ $\mathbb{R}^{n(N+1)}$ is a controlled $(k, \lambda)$-contractive set. In addition, set-induced state-feedback control laws can be established by computing input sequences which drive all trajectories starting from the vertices of the set $\mathcal{Z}$ in $\lambda \mathcal{Z}$ after $k$ time steps. Indeed, let $\mathcal{Z} \subset \mathbb{R}^{n(N+1)}$,

$$
\mathcal{Z}:=\operatorname{conv}\left(\left\{v_{z}^{i}\right\}_{i \in \mathbb{N}_{[1, q z]}}\right),
$$

be an arbitrary proper $\mathcal{C}$-polytopic set and let $V_{z, 0} \in$ $\mathbb{R}^{n(N+1) \times q_{z}}$ denote the full-row rank matrix that has as columns the vertices $v_{z}^{i} \in \mathbb{R}^{n(N+1)}, i \in \mathbb{N}_{\left[1, q_{z}\right]}$.

Problem 1 Consider the discrete-time linear system (4) and the controlled $(k, \lambda)$-contractive set $\mathcal{Z}$, defined in (10). For each $l \in \mathbb{N}_{\left[1, q_{z}\right]}$, solve the following $q_{z}$ feasibility problems, setting $v_{z, 0}^{l}:=v_{z}^{l}$.

$$
\text { find }\left\{u_{z, i}^{l}\right\}_{i \in \mathbb{N}_{[0, k-1]}},\left\{v_{z, i}^{l}\right\}_{i \in \mathbb{N}_{[1, k]}}, p^{l}
$$

subject to

$$
\begin{aligned}
v_{z, i+1}^{l} & =A_{z} v_{z, i}^{l}+B_{z} u_{z, i}^{l}, \forall i \in \mathbb{N}_{[0, k-1]}, \\
v_{z, k}^{l} & =V_{z, 0} p^{l}, \\
p^{l} & \geq 0_{q_{z}}, \\
1_{q}^{\top} p^{l} & \leq \lambda .
\end{aligned}
$$

Let $U_{z, i} \in \mathbb{R}^{m \times q_{z}}, i \in \mathbb{N}_{[0, k-1]}, V_{z, i} \in \mathbb{R}^{n \times q}, i \in \mathbb{N}_{[1, k]}$ be the matrices constructed from the solution of Problem 1 in the following manner

$$
\begin{aligned}
& {\left[U_{z, i}\right]_{: j}:=u_{z, i}^{j}, \quad(i, j) \in \mathbb{N}_{[0, k-1]} \times \mathbb{N}_{\left[1, q_{z}\right]},} \\
& {\left[V_{z, i}\right]_{: j}:=v_{z, i}^{j}, \quad(i, j) \in \mathbb{N}_{[1, k-1]} \times \mathbb{N}_{\left[1, q_{z}\right]} .}
\end{aligned}
$$

Consider the state-feedback control law $g(\cdot): \mathcal{Z} \rightarrow \mathbb{R}^{m}$

$$
g\left(z_{t}\right):=\pi_{i}\left(z_{t}\right) \quad \text { if } t=k M+i, M \in \mathbb{N}
$$

where

$$
\pi_{i}\left(z_{t}\right):=U_{z, i} \mu_{i}\left(z_{t}\right)
$$

for all $i \in \mathbb{N}_{[0, k-1]}, \mu_{i}\left(z_{t}\right) \in \mathcal{M}_{i}\left(z_{t}\right)$,

$$
\begin{aligned}
& \mathcal{M}_{0}\left(z_{t}\right):=\left\{\mu \in \mathbb{R}_{+}^{q_{z}}: z_{t}=V_{0} \mu, 1_{q}^{\top} \mu \leq 1\right\} \\
& \mathcal{M}_{i}\left(z_{t}\right):=\left\{\mu \in \mathbb{R}_{+}^{q_{z}}: V_{z, i} \mu=V_{z, i} \mu_{i-1}\left(z_{t}\right), 1_{q}^{\top} \mu \leq 1\right\} .
\end{aligned}
$$

Proposition 2 [22, Proposition 3]. The system (7) under the set-valued control law (13)-(16) is $\mathcal{K} \mathcal{L}$-stable in $\mathcal{Z}$.

Fact 4 The map $g(\cdot): \mathcal{Z} \rightarrow \mathbb{R}^{m}$, defined in (13)-(16), is homogeneous in $\mathcal{Z}$.

Fact 4 is derived by observing that the mappings $\pi_{i}(\cdot), i \in$ $\mathbb{N}_{[0, k-1]}$, as defined in (14), are homogeneous in $\mathcal{Z}$. The 
following results follow directly from Proposition 1, Fact 3, Proposition 2 and Fact 4.

Theorem 1 Let the set $\mathcal{Z} \subset \mathbb{R}^{n(N+1)}$, defined in (10), be a controlled $(k, \lambda)$-contractive set. Consider the control law $g(\cdot): \mathcal{Z} \rightarrow \mathbb{R}^{m}$ (13)-(16), and the control law $\bar{g}(\cdot):$ $\mathbb{R}^{n(N+1)} \rightarrow \mathbb{R}^{m}$, where

$$
\bar{g}\left(z_{t}\right)=\Psi\left(\mathcal{Z}, z_{k M}\right) g\left(\Psi^{-1}\left(\mathcal{Z}, z_{k M}\right) z_{t}\right), \quad \text { if } t=k M+i,
$$

for all $z_{k M} \in \mathbb{R}^{n(N+1)} \backslash\{0\}$, and $\bar{g}(0):=0$. Then, the closed-loop system $z_{t+1}=A_{z} z_{t}+B_{z} \bar{g}\left(z_{t}\right)$ is $\mathcal{K} \mathcal{L}$-stable in $\mathbb{R}^{n(N+1)}$.

Proposition 3 Let the set $\mathcal{Z} \subset \mathbb{R}^{n(N+1)}$ be a controlled $(k, \lambda)$-contractive set for (4). Then, for all $\alpha \in \mathbb{R}_{+}, \alpha \mathcal{Z}$ is a controlled $(k, \lambda)$-contractive set for (4).

\section{B. Characterization of controlled $(k, \lambda)-D$-contractive sets}

In this section we establish two families of proper $\mathcal{C}-$ polytopic controlled $(k, \lambda)$-contractive sets defined in the augmented state space $\mathbb{R}^{n(N+1)}$, which induce controlled $(k, \lambda)-D$ contractive sets in the original state space of the time-delay system. To this end, given a proper $\mathcal{C}$-polytopic set $\mathcal{S} \subset \mathbb{R}^{n}$ with vertex representation (1), we first consider the family of sets $\mathcal{Z}_{\text {inf }}(\mathcal{S}) \subset \mathbb{R}^{n(N+1)}$, where

$$
\mathcal{Z}_{\text {inf }}(\mathcal{S}):=\left\{z \in \mathbb{R}^{n(N+1)}: \max _{i \in \mathbb{N}_{[1, N+1]}} \Psi\left(\mathcal{S},[z]_{i}\right) \leq 1\right\} .
$$

The set $\mathcal{Z}_{\text {inf }}(\mathcal{S}) \subset \mathbb{R}^{n(N+1)}$ represents the $N+1$ Cartesian product of the set $\mathcal{S}$. It has $q^{(N+1)}$ vertices, which are denoted by $v_{z}^{l}, l \in \mathbb{N}_{\left[1, q^{(N+1)}\right]}$. We remind that $q \in \mathbb{N}$ is the number of vertices of the set $\mathcal{S} \subset \mathbb{R}^{n}$. Let $V_{z, 0} \in$ $\mathbb{R}^{n(N+1) \times q^{(N+1)}}$, where $\left[V_{z, 0}\right]_{: l}=v_{z}^{l}, l \in \mathbb{N}_{\left[1, q^{(N+1)}\right]}$, be the full row rank matrix having as columns the vertices of $\mathcal{Z}_{\text {inf }}(\mathcal{S})$. The next result follows directly.

Proposition 4 Let $\mathcal{Z}_{\text {inf }}(\mathcal{S})$ be a controlled $(k, \lambda)$-contractive set with respect to system (4). Then, $\mathcal{S}$ is a controlled $(k, \lambda)-$ $D$-contractive set with respect to system (2).

A stabilizing controller can be constructed by solving Problem 1 for the vertices of the set $\mathcal{Z}_{\text {inf }}(\mathcal{S})$. Nevertheless, the implementation of the corresponding control strategy (13)(16) is not scalable with respect to the maximum delay $N$. In specific, the computation of the vectors $\mu_{i}\left(\boldsymbol{x}_{[t-N, t]}\right) \in$ $\mathcal{M}_{i}\left(\boldsymbol{x}_{[t-N, t]}\right)$ requires the decomposition of the augmented vector $z_{t}$ constructed from the states $\boldsymbol{x}_{[t-N, t]}$ to the convex combination of the $q^{N+1}$ vertices of $\mathcal{Z}_{\text {inf }}(\mathcal{S})$. To tackle this problem, we consider the family of sets $\mathcal{Z}_{1}(\mathcal{S}) \subset \mathbb{R}^{n(N+1)}$, where

$$
\mathcal{Z}_{1}(\mathcal{S}):=\left\{z \in \mathbb{R}^{n(N+1)}: \sum_{i=1}^{N+1} \Psi\left(\mathcal{S},[z]_{i}\right) \leq 1\right\} .
$$

For any proper $\mathcal{C}$-polytopic set $\mathcal{S} \subset \mathbb{R}^{n}, \mathcal{Z}_{1}(\mathcal{S}) \subset \mathbb{R}^{n(N+1)}$ is also a proper $\mathcal{C}$-polytopic set and has $q(N+1)$ vertices.
The next results follow from the properties of Minkowski functions and the structure of the sets $\mathcal{Z}_{\text {inf }}(18)$ and $\mathcal{Z}_{1}(19)$.

Fact 5 For any proper $\mathcal{C}$-set $\mathcal{S} \subset \mathbb{R}^{n}$, it holds that

$$
\mathcal{Z}_{1}(\mathcal{S}) \subseteq \mathcal{Z}_{\text {inf }}(\mathcal{S}) \subseteq(N+1) \mathcal{Z}_{1}(\mathcal{S})
$$

Proposition 5 Let $\mathcal{Z}_{1}(\mathcal{S})$ be a controlled $(k, \lambda)$-contractive set with respect to system (4). Then, $\mathcal{S}$ is a controlled $\left(k^{\star}, \lambda\right)-$ $D$-contractive set with respect to the system (2), with

$$
k^{\star}=\left\lceil 1-\frac{\log (N+1)}{\log \lambda}\right\rceil k \text {. }
$$

Comparing Propositions 4 and 5 it is worth noticing that the computations needed to characterize the set $\mathcal{S}$ as a controlled $(k, \lambda)-D$-contractive set are significantly less using the results of Proposition 5. In detail, the set $\mathcal{Z}_{1}(\mathcal{S})$ consists of $q(N+1)$ vertices. Thus, characterizing the set $\mathcal{Z}_{1}$ as a controlled $(k, \lambda)$-contractive set is less computationally expensive than characterizing the set $\mathcal{Z}_{\text {inf }}(\mathcal{S})$, which consists of $q^{(N+1)}$ vertices.

\section{Scalable controller synthesis and implementation}

In what follows, a systematic scalable controller synthesis and implementation method is provided. Consider the proper $\mathcal{C}$-polytopic set $\mathcal{S}(1)$ and the initial condition sequences for the system (2), denoted by $\boldsymbol{v}_{i[-N, 0]}^{l}:=\left\{v_{i, j}^{l}\right\}_{j \in \mathbb{N}_{[-N, 0]}}$, for all $(i, l) \in \mathbb{N}_{[-N, 0]} \times \mathbb{N}_{[1, q]}$, where

$$
v_{i, j}^{l}:=\left\{\begin{array}{lr}
v^{l}, & j=i, \\
0_{n}, & j \in \mathbb{N}_{[-N, 0]} \backslash\{i\} .
\end{array}\right.
$$

We consider the following problem.

Problem 2 Consider the linear time-delay system (2) and the proper $\mathcal{C}$-polytopic set $\mathcal{S}(1)$. Solve the following $q(N+$ 1) feasibility problems, for each pair $(i, l) \in \mathbb{N}_{[-N, 0]} \times \mathbb{N}_{[1, q]}$.

$$
\text { find }\left\{v_{i, j}^{l}\right\}_{j \in \mathbb{N}_{[1, k]}},\left\{u_{i, j}^{l}\right\}_{j \in \mathbb{N}_{[0, k-1]}},\left\{p_{i, j}^{l}\right\}_{j \in \mathbb{N}_{[-N, 0]}}
$$

subject to

$$
\begin{gathered}
v_{i, j+1}^{l}=\sum_{c=0}^{N} A_{i} v_{i, j-c}^{l}+B u_{i, j}^{l}, \quad j \in \mathbb{N}_{[0, k-1]}, \\
v_{i, k+j}^{l}=V p_{i, j}^{l}, \quad j \in \mathbb{N}_{[-N, 0]}, \\
p_{i, j}^{l} \geq 0_{q}, \quad j \in \mathbb{N}_{[-N, 0]}, \\
\sum_{j=-N}^{0} 1_{q}^{\top} p_{i, j}^{l} \leq \lambda .
\end{gathered}
$$

Suppose that the Problem 2 is feasible and consider the

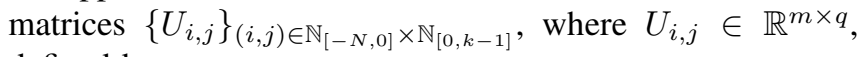
defined by

$$
\left[U_{i, j}\right]_{: l}:=u_{i, j}^{l}, \quad l \in \mathbb{N}_{[1, q]} .
$$

We define the control law

$$
g_{x}\left(\boldsymbol{x}_{[t-N, t]}\right):=\sum_{i=-N}^{0} U_{i, j} \mu\left(x_{k M+i}\right), \text { if } t=k M+j,
$$


where $\mu(x) \in \mathcal{M}(x)$,

$$
\mathcal{M}(x):=\left\{\mu \in \mathbb{R}_{+}^{q}: x=V \mu\right\} .
$$

Next, we establish that the problem of characterizing the set $\mathcal{S}$ as a controlled $(k, \lambda)-D$-contractive set and computing a stabilizing control law for system (2) is scalable.

Theorem 2 Suppose that Problem 2 admits a feasible solution. Then, the following hold.

(i) The proper $\mathcal{C}$-polytopic set $\mathcal{S}(1)$ is a controlled $\left(k^{\star}, \lambda\right)$ $D$-contractive set with respect to (2).

(ii) The closed-loop system (6) under the control law

$$
\begin{aligned}
g\left(\boldsymbol{x}_{[t-N, t]}\right) & :=\gamma\left(\boldsymbol{x}_{[k M-N, k M]}\right) \times \\
& g_{x}\left(\gamma\left(\boldsymbol{x}_{[k M-N, k M]}\right)^{-1} \boldsymbol{x}_{[t-N, t]}\right)
\end{aligned}
$$

if $t=k M+i$, where $g_{x}(\cdot): \mathbb{R}^{n(N+1)} \rightarrow \mathbb{R}^{m}$ is defined in (28), (29) and

$$
\gamma\left(\boldsymbol{x}_{[k M-N, k M]}\right):=\sum_{i=-N}^{0} \Psi\left(\mathcal{S}, x_{k M+i}\right),
$$

is globally $\mathcal{K} \mathcal{L}-$ stable.

Proof: (i) Consider the vectors $\left\{v_{z, 0}^{l}\right\}_{l \in \mathbb{N}_{1, q(N+1)}}$, $v_{z, 0}^{l} \in \mathbb{R}^{n(N+1)}$ of initial conditions in the augmented space $\mathbb{R}^{n(N+1)}$, where $\left[v_{z, 0}^{(1-i) c}\right]_{1-j}:=v_{i, j}^{c}, \quad(i, c, j) \in \mathbb{N}_{[-N, 0]} \times$ $\mathbb{N}_{[1, q]} \times \mathbb{N}_{[-N, 0]}$. The vectors $v_{z, 0}^{l}$ are the vertices of the set $\mathcal{Z}_{1}(\mathcal{S})(19)$. It is easy to verify that relations (12) are satisfied with $u_{z, j}^{(1-i) c}:=u_{i, j}^{c},(i, c, j) \in \mathbb{N}_{[-N, 0]} \times \mathbb{N}_{[1, q]} \times \mathbb{N}_{[0, k-1]}$, and $\left[p_{z}^{(1-i) c}\right]_{j}:=p_{i, j}^{c}, j \in \mathbb{N}_{[-N, 0]}$. Since Problem 1 has a feasible solution, the set $\mathcal{Z}_{1}(\mathcal{S})$ is a controlled $(k, \lambda)-$ contractive set with respect to (4). Consequently, from Proposition 5, it follows that $\mathcal{S}$ is a controlled $\left(k^{\star}, \lambda\right)-D$ contractive set, with $k^{\star}=\left\lceil 1-\frac{\log (N+1)}{\log \lambda}\right\rceil k$.

(ii) Since $\mathcal{Z}_{1}(\mathcal{S})$ is a controlled $(k, \lambda)$-contractive set, it follows that the control law (13)-(16) is stabilizing for all $z_{0} \in \mathcal{Z}_{1}(\mathcal{S})$. Consider the explicit admissible selection $\mu_{i}\left(z_{t}\right):=\mu_{0}\left(z_{t}\right)$, for all $i \in \mathbb{N}_{[1, k-1]}$ of the setvalued law (13)-(16). The corresponding control law is $g_{z}\left(z_{t}\right):=U_{z, i} \mu\left(z_{t}\right)$, if $t=k M+i, M \in \mathbb{N}$, where $U_{z, j}=\left[\begin{array}{llll}U_{0, j} & U_{-1, j} & \cdots & U_{-N, j}\end{array}\right]$, for all $j \in \mathbb{N}_{[0, k-1]}$, with $\mu\left(z_{t}\right) \in \mathcal{M}_{z}\left(z_{t}\right) \subset \mathbb{R}^{q}$, where $\mathcal{M}_{z}(z):=\{\mu \in$ $\left.\mathbb{R}_{+}^{q(N+1)}: z=V_{z, 0} \mu\right\}$. The matrix $V_{z, 0} \in \mathbb{R}^{n(N+1) \times q(N+1)}$ contains in its columns the vertices of the set $\mathcal{Z}_{1}(\mathcal{S})$ and is equal to $V_{z, 0}=\left[\begin{array}{cccc}V & 0_{n \times q} & \cdots & 0_{n \times q} \\ 0_{n \times q} & V & \cdots & 0_{n \times q} \\ \vdots & \vdots & \ddots & \vdots \\ 0_{n \times q} & 0_{n \times q} & \cdots & V\end{array}\right]$. Thus, it follows that the set $\mathcal{M}_{z}(z)$ is equal to $\mathcal{M}_{z}(z)=\left\{\mu \in \mathbb{R}^{q(N+1)}\right.$ : $\left.[z]_{i}=V[\mu]_{i}, i \in \mathbb{N}_{[1, N+1]}\right\}$. Consequently, the control law can be written as $g_{z}\left(z_{t}\right):=\sum_{j=1}^{N+1} U_{(1-j), i}\left[\mu\left(z_{t}\right)\right]_{j}$, if $t=$ $k M+i, M \in \mathbb{N}$, and taking into account (3), it is equal to (28), (29). From Proposition 2 and [5], it follows that the closed-loop system (6) under the control law (28), (29) is $\mathcal{K} \mathcal{L}$-stable in $\mathcal{S}$. Taking into account Theorem 1 and that relation $\Psi\left(\mathcal{Z}_{1}, z\right)=\sum_{i=1}^{N+1} \Psi\left(\mathcal{S},[z]_{i}\right)$ holds by construction of the set $\mathcal{Z}_{1}(\mathcal{S})$, it follows directly that the closed-loop system (6) under the control law (30) is globally $\mathcal{K} \mathcal{L}$-stable.

Remark 1 The first statement of Theorem 2 establishes scalability of the computations needed to characterize a proper $\mathcal{C}$-polytopic set $\mathcal{S}$ as a controlled $(k, \lambda)-D$-contractive set. Indeed, solving Problem 2 with initial condition vector sequences $\boldsymbol{v}_{\boldsymbol{i}[-N, 0]}^{l}$, defined by (22), is equivalent to solving Problem 1, defined in the augmented state space, with initial conditions the vertices of the set $\mathcal{Z}_{1}(\mathcal{S})$. Moreover, each of the $q(N+1)$ linear programs in Problem 2 is already in a form such that it can be solved using distributed optimization techniques, see for example [23, Section 4.2].

Remark 2 The second statement of Theorem 2 establishes a globally stabilizing scalable state-feedback control law. In detail, the control law (30) can be implemented as follows: Given the time variable $t$, find the integers $(M, i) \in$ $\mathbb{N} \times \mathbb{N}_{[0, k-1]}$ such that $t=k M+i$. If $i=0$, compute $\Psi\left(\mathcal{S}, x_{k M+j}\right)$, for all $j \in \mathbb{N}_{[-N, 0]}{ }^{1}$ and $\gamma\left(\boldsymbol{x}_{[k M-N, k M]}\right)$, as defined in (31). Next, compute $\mu\left(\gamma\left(\boldsymbol{x}_{[k M-N, k M]}\right)^{-1} x_{k M+j}\right)$, for all $j \in \mathbb{N}_{[-N, 0]}$, as defined in (29), and $g\left(\boldsymbol{x}_{[t-N, t]}\right)$, as defined in (30). If $i \in \mathbb{N}_{[1, k-1]}$, compute $g\left(\boldsymbol{x}_{[t-N, t]}\right)$, as defined in (30).

\section{NUMERICAL EXAMPLE}

We consider a second order time-delay system (2) with maximum delay $N=4$. The system matrices $A_{i} \in$ $\mathbb{R}^{2 \times 2}, i \in \mathbb{N}_{[0,4]}$, and $B \in \mathbb{R}^{2 \times 1}$ are $A_{0}=\left[\begin{array}{cc}1.5 & -0.5 \\ 0.5 & 0.2\end{array}\right]$, $A_{1}=\left[\begin{array}{cc}0.2 & -0.2 \\ 0.2 & 0.2\end{array}\right], A_{2}=\left[\begin{array}{cc}0.5 & 0.5 \\ 0.5 & 0.2\end{array}\right], A_{3}=\left[\begin{array}{cc}-0.6 & 0.3 \\ 1 & 2\end{array}\right], A_{4}=$ $\left[\begin{array}{cc}1.5 & -0.5 \\ 0.5 & 0.2\end{array}\right], B=\left[\begin{array}{l}1 \\ 1\end{array}\right]$. For the given numerical example, we applied two standard approaches for constructing a stabilizing state-feedback controller and a region of attraction.

First, a controlled invariant set $\mathcal{Z} \subset \mathbb{R}^{n(N+1)}$ was searched for, for the augmented system $\left(A_{z}, B_{z}\right), A_{z} \in$ $\mathbb{R}^{10 \times 10}, B_{z} \in \mathbb{R}^{10 \times 1}$. This approach belongs to the family of Lyapunov-Krasovskii methods, since the controlled invariant set is searched in the augmented space. The algorithm for finding the controlled invariant set was implemented by the routine mpt_maxCtrlset of the MPT [24]. The algorithm did not succeed in returning a solution. Next, we aimed to find a $D$-invariant set $\mathcal{S} \subset \mathbb{R}^{2}$ by exploiting the necessary and sufficient algebraic conditions of existence of polyhedral $D$-invariant sets [17]. This approach belongs to the family of Lyapunov-Razumikhin methods. To this end, the discrete LQR control was computed for the augmented system. We attempted to solve the algebraic conditions for the closed-loop time-delay system using the fmincon routine of Matlab. For this setting, no feasible solution could be obtained, for candidate polytopic $D$-invariant sets which can be described as the intersection of at most 40 half-spaces, while no solution was returned for more complex candidate polytopic sets. The failure of the considered standard approaches indicates the non-trivial nature of the stabilization problem

\footnotetext{
${ }^{1}$ Since $\mathcal{S}$ is a proper $\mathcal{C}$-polytopic set, $\Psi(\mathcal{S}, x)$ is equal to the optimal cost of the linear program $\min _{\alpha, \xi} \alpha$ subject to $x=V \xi$ and $1_{q}^{\top} \xi \leq \alpha$.
} 


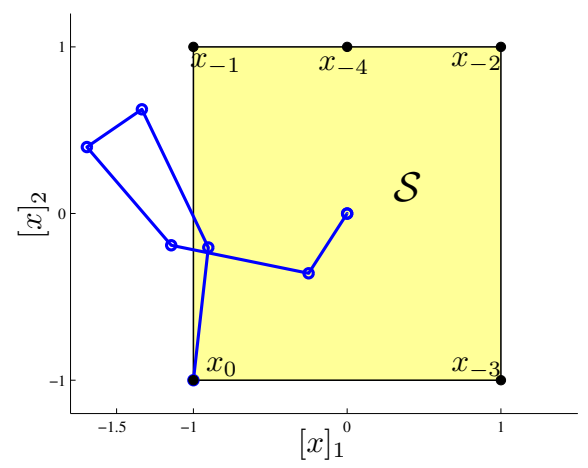

Fig. 1. The $\left(k^{\star}, \lambda\right)-D$-contractive set $S$ (yellow), the initial conditions $x_{-4}=\left[\begin{array}{ll}0 & 1\end{array}\right]^{\top}, x_{-3}=\left[\begin{array}{ll}1 & -1\end{array}\right]^{\top}, x_{-2}=\left[\begin{array}{ll}1 & 1\end{array}\right]^{\top}, x_{-1}=\left[\begin{array}{ll}-1 & 1\end{array}\right]^{\top}$, $x_{0}=\left[\begin{array}{ll}-1 & -1\end{array}\right]^{\top}$ (black dots) and the trajectory of the closed-loop system (6) under the control law (30), (31) (blue circles).

for the numerical example under study. In what follows, we demonstrate the method proposed in this article for the same example. In detail, the problem investigated concerns the characterization of the initial condition set $\mathcal{S}:=\left\{x \in \mathbb{R}^{2}\right.$ : $\left.\|x\|_{\infty} \leq 1\right\}$ as a candidate $(k, \lambda)-D$-contractive set and the determination of a stabilizing state-feedback control law for all $x_{i} \in \mathcal{S}, i \in \mathbb{N}_{[-4,0]}$. The set $\mathcal{S}$ has four vertices $v^{i}, i \in \mathbb{N}_{[1,4]}$, where $v^{1}=\left[\begin{array}{ll}1 & 1\end{array}\right]^{\top}, v^{2}=\left[\begin{array}{ll}-1 & 1\end{array}\right]^{\top}$, $v^{3}=\left[\begin{array}{ll}1 & -1\end{array}\right]^{\top}, v^{4}=\left[\begin{array}{ll}-1 & -1\end{array}\right]^{\top}$. First, the set $\mathcal{S}$ was characterized as a $(k, \lambda)-D$-contractive set by exploiting Theorem 2. In specific, a pair $(k, \lambda) \in \mathbb{N}_{\geq 1} \times \mathbb{R}_{[0,1)}$ was computed such that Problem 2 has a solution. The integer $k$ was increased until a feasible solution was recovered. The feasible integer $k=10 \in \mathbb{N}_{\geq 1}$ and scalar $\lambda=2 \times 10^{-31} \in$ $\mathbb{R}_{[0,1)}$ were obtained by solving the optimization problem

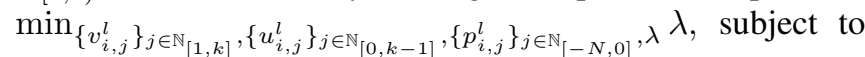
the constraints (24)-(27) and $0 \leq \lambda<1$, for $q(N+1)=$ $4 \times 5=20$ different initial condition sequences $\boldsymbol{v}_{\boldsymbol{i}[-N, 0]}^{l}$, as defined in (22). Thus, from Theorem 2 , the set $\mathcal{S}$ is a $\left(k^{\star}, \lambda\right)-D$-contractive set with respect to the system (2), where $k^{\star}=\left[1-\frac{\log (5)}{\log \left(2 \times 10^{-31}\right)}\right] 10=20$. The control law (30),(31) is a globally stabilizing state-feedback control law, thus, it is stabilizing for all $x_{i} \in \mathcal{S}, i \in \mathbb{N}_{[-4,0]}$. In Figure 1, the $\left(20,2 \times 10^{-31}\right)-D$-contractive set $\mathcal{S}$ is shown with yellow color in the time-delay state space. Moreover, a trajectory of the closed-loop system under the control law (30), (31) is shown in blue color, for initial conditions $x_{i} \in \mathcal{S}, i \in \mathbb{N}_{[-4,0]}$, which are shown as black dots.

\section{CONCLUSions}

A novel stabilizing controller synthesis and implementation approach for discrete-time linear time-delay systems was presented. The proposed approach is non-conservative, it is scalable with respect to the maximum delay, and it induces controlled $(k, \lambda)-D$-contractive sets, which correspond to a relaxed notion of invariance in the time-delay space.

\section{REFERENCES}

[1] V. Kharitonov, "Robust stability analysis of time delay systems: A survey," Annual Reviews in Control, vol. 23, pp. 185-196, 1999.
[2] J. P. Richard, "Time-delay systems: an overview of some recent advances and open problems," Automatica, vol. 39, pp. 1667-1694, 2003

[3] R. Sipahi, S. I. Niculescu, C. T. Abdallah, W. Michiels, and K. Gu, "Stability and stabilization of systems with time delay - limitations and opportunities," IEEE Control Systems Magazine, vol. 31, pp. 38-65, 2011.

[4] W. Lombardi, "Constrained control for Time-Delay Systems," Ph.D. dissertation, Supelec, 2011.

[5] R. H. Gielen, "Stability Analysis and Control of Discrete-Time Systems with Delay," Ph.D. dissertation, Eindhoven University of Technology, 2013.

[6] K. J. Astrom and B. Wittenmark, Computer controlled systems, theory and design. Englewood Cliffs, NJ, Prentice Hall International, 1990

[7] E. Fridman, A. Seuret, and J. P. Richard, "Robust sampled-data stabilization of linear systems: an input delay approach," Automatica, vol. 40, pp. 1441-1446, 2004.

[8] W. Lombardi, S. Olaru, S. I. Niculescu, and L. Hetel, "A predictive control scheme for systems with variable time-delay," International Journal of Control, vol. 85, pp. 915-932, 2012.

[9] M. B. G. Cloosterman, L. Hetel, N. van de Wouw, W. P. M. H Heemels, J. Daafouz, and H. Nijmeijer, "Controller synthesis for networked control systems," Automatica, vol. 46, pp. 1584-1594, 2010.

[10] B. Liu and H. J. Marquez, "Razumikhin-type stability theorems for discrete delay systems," Automatica, vol. 43, pp. 1219-1225, 2007.

[11] Z. Lin, "On Asymptotic Stabilizability of Discrete-Time Linear Systems with Delayed Input," in IEEE International Conference in Control and Automation, Guangzhou, China, 2007, pp. 432-437.

[12] R. H. Gielen and M. Lazar, "Stabilization of polytopic delay difference inclusions via the Razumikhin approach," Automatica, vol. 47, pp. 2562-2570, 2011

[13] G. Bitsoris, N. Athanasopoulos, and L. Dritsas, "Stability, positive invariance and design of constrained regulators for networked control systems," International Journal of Control, vol. 85, pp. 1401-1413, 2012.

[14] R. Gielen, M. Lazar, and S. Rakovic, "Necessary and sufficient Razumikhin-type conditions for stability of delay difference equations," IEEE Transactions on Automatic Control, vol. 58, pp. 26372642, 2013.

[15] J. K. Hale and S. M. V. Lunel, Introduction to Functional Differential Equations. Springer-Verlag, 1993.

[16] R. J. P. Dambrine and P. Borne, "Feedback control of time-delay systems with bounded control and state," Mathematical Problems in Engineering, vol. 1, pp. 77-87, 1995.

[17] J. Hennet and S. Tarbouriech, "Stability conditions of constrained delay systems via positive invariance," International Journal of Robust and Nonlinear Control, vol. 8, pp. 265-278, 1998.

[18] W. Lombardi, S. Olaru, M. Lazar, and S. I. Niculescu, "On Positive Invariance for Delay Difference Equations," in 18th IFAC World Congress, Milano, Italy, 2011, pp. 3674-3679.

[19] W. Lombardi, S. Olaru, G. Bitsoris, and S. I. Niculescu, "Cyclic invariance for discrete-time delay systems," Automatica, vol. 48, pp. 2730-2733, 2012

[20] S. V. Rakovic, R. H. Gielen, and M. Lazar, "Construction of invariant families of sets for linear systems with delay," in American Control Conference, Montreal, Canada, 2012, pp. 6246-6251.

[21] M. Lazar, A. I. Doban, and N. Athanasopoulos, "On stability analysis of discrete-time homogeneous dynamics," in 17th International Conference on System Theory, Control and Computing, Sinaia, Romania, 2013, pp. 297-305.

[22] N. Athanasopoulos, A. I. Doban, and M. Lazar, "On Constrained Stabilization of Discrete-Time Linear Systems," in 21st Mediterranean Conference on Control and Automation (MED), Chania, Greece, 2013, pp. 831-839.

[23] N. Athanasopoulos and M. Lazar, "Scalable Stabilization of LargeScale Discrete-Time Linear Systems via the 1-norm," in 4th IFAC Workshop on Distributed Estimation and Control in Networked Systems, Koblenz, Germany, 2013, pp. 277-284.

[24] M. Kvasnica, P. Grieder, and M. Baotic, M. amd Morari, "MultiParametric Toolbox," in 7th International Workshop on Hybrid Systems: Computation and Control, Philadelphia, USA, 2004, pp. 448462. 